\title{
Role of Filler-Polymer Interface on the Thermal Conductivity in Polymer Composites
}

\author{
Vijendra Kumar', Abhishek Barnwal', R.K. Shukla ${ }^{3}$, Jyoti Shakya ${ }^{4, *}$ \\ ${ }^{1} \mathrm{PhD}$ Student, Department of Physics, University of Lucknow, Lucknow Uttar Pradesh, India \\ ${ }^{2}$ Research Scholar, Department of Chemical Engineering, Indian Institute of Technology Delhi, \\ New Delhi, India \\ ${ }^{3}$ Professor, Department of Physics, University of Lucknow, Lucknow, Uttar Pradesh, India \\ ${ }^{4}$ Post-doctoral Research Fellow, Department of Physical Sciences, Indian Institute of Sciences, \\ Bangalore, Karnataka, India
}

\begin{abstract}
Polymers with high thermal conductivity are the need of modern technologies due to their robustness, cost-effectiveness and less corrosiveness. However, bare polymers are not good heat-conductors due to their molecular structures, and their non-metallic properties. Therefore, metallic fillers of different shapes and sizes have been used to enhance the thermal conductivity in polymer-composites. However, there has been search for best geometrical fillers which can maximize the thermal conductivity effectively with same volume percent. From our numerical simulations, we show that the best geometrical fillers are those which have high surface-area $(S)$ to volume $(V)$ ratio. In such cases, the interface of polymer-filler is maximized, which leads to the effective enhancement in the thermal conductivity. To validate our claim, we use fillers with same volume percent but maximize the surface area. We also show that there is a competition between surface and the bulk of the filler in this maximization process but surface-area dominates with an effective increase in the thermal conductivity. We use 3-dimensional models using ANSYS-Fluent to show the characteristic behaviour of thermal conductivity. Polyethylene has been used as the base polymer and aluminium (Al) has been used as filler in all our model simulation.
\end{abstract}

Keywords: Polymer composites, fillers, 3-dimensional models, thermal conductivity, polyethylene, aluminium (Al)

*Author for Correspondence E-mail: jyiitd@gmail.com

\section{INTRODUCTION}

Composite materials are the host of new technologies. They constitute the new building blocks of engineering and modern scientific advancement. The most studied composite materials are the composite polymers. Polymers have played an important role in the industrial development [1-3] and numerous biomedical applications due to their low-cost fabrication, robustness, corrosion resistance and due to their light-weight $[4,5]$. Polymers exist in our day to day life and have facilitated the human society from the very beginning of their discoveries. Now they are being used in many mechanical and electrical appliances. Polymers are also being used as heat exchangers in various cases such as chemical processing units, refrigeration, air conditioning, food and beverage industry, liquid desiccant cooling system, micro-scale cooling systems, electronic cooling devices, seawater desalination plants, microelectronic devices, mechanical heat units etc. [6-8].

However, the composite polymers have limitations as heat exchangers because of their low thermal conductivity. The thermal conductivity of polymers is very low, and at least 100-200 times lower than the conventional metals such as iron, copper, aluminium etc. [9-11]. In spite of these limitations, there has been search of more functional composite materials in the form of composite polymers which should have high thermal conductivity. Note that the composites are the materials which are composed of 
different phases. Each phase corresponds to a homogeneous and uniform system, i.e., a new component, or a new material. These constituent phases have their unique physical and chemical properties, and the characteristic properties for one phase may be strikingly different from the others. However, the composite may have properties very different from the individual components. According to the general definition, composites constitute more than $5 \%$ of each phase, but there is no as such any hard and fast rule. For example, in most of the chemical processes and in chemical engineering applications, there is need of composite polymers to avoid any corrosion due to various chemical reactions. In such applications, metals cannot have much volume percentage as they may decompose under the acidic reactions, leading to the destruction of the whole processing unit. In these cases, the polymer composites with low volume fraction of metals are the best option to avoid any decomposition [12-15].

One of the basic questions that arise is that why the thermal conductivity of polymers is low? The fundamental reason behind this is the molecular structure of the polymer itself. We know that polymers are made of monomers by the polymerization process. These structures are such that they restrict the propagation of heat carrier phonons. In crystals on the other hand, phonons can propagate for the larger distances without any hindrance and they propagate linearly. In polymers, each individual molecule works as heat processing unit. Therefore, the process of heat conduction polymers is different than crystals [16-18].

There has been extensive research in the design and fabrication of polymer composites in the perspective of obtaining good heat conductors. Most of these researches are based on the composites made of two components, i.e., matrix polymer and metallic fillers. It has been observed that the effective thermal conductivity varies with the shape and size of the fillers. Therefore, fillers of various shapes and sizes have been used to maximize the thermal conduction [19-21]. Still the basic question remains the same. What are the best shapes and what are the best geometries to maximize the thermal conductivity in these composite polymers. Further, it is interesting to know why the filler of a particular shape or geometry results in maximization of thermal conductivity than the other shapes or the geometries.

There has not been any satisfactory answer to this question. In this study we try to answer this very basic question based on our numerical simulations. We have done extensive numerical simulation to propose that it is the surface area of the fillers which can maximize the effective thermal conductivity. Keeping the volume percent of the filler constant, and maximizing its surface area we can maximize the interface between the polymer and the filler, and this is the basic reason behind the effective enhancement of the thermal conductivity. This can be understood in the view of polymer structure itself. As mentioned earlier, each molecule of the polymer works as heat processing unit, and when the surface area of the filler is maximized, the interface contact area between the polymer and filler is also maximized [22-25]. This causes more polymer molecules to come in to contact with the metallic filler. This gives rise to more heat processing units in the form of polymer molecules and thus leading to effectively enhancing the thermal conductivity. Therefore, the basic mechanism for enhancing the thermal conductivity in the composite polymers is the surface maximization of the fillers used in various shapes [26, 27]. We have used the ANSYSfluent 18.0 software. This paper is organized as follows: In the next part of the paper we describe in detail the governing heat-flux equations, and their applicability in polymer composites. Then we give detailed results and discussion of our numerical simulations and finally, we conclude the paper with a brief summary.

\section{HEAT TRANSFER EQUATIONS}

In this section, we describe the equations governing the heat transfer in 3-dimensional geometries. In Figure 1 we show a schematic diagram which has been used as our model system to describe the flow of heat from the 
top to the bottom surface in the compositepolymer systems considered in the present manuscript. The heat-flux is constrained in only a single direction (i.e., from top to bottom surface or from left to the right surface), and the corresponding heat equations are modified with the different boundary conditions.

As shown in Figure 1, in the generation of the three-dimensional cubic cell, the heat transfer in the base or at the bottom surface of the polymer matrix is satisfied by the following equation:

$$
\frac{K_{p}}{\left(\rho c_{p}\right)_{p}}\left[\frac{\partial^{2} T_{p}}{\partial x^{2}}+\frac{\partial^{2} T_{p}}{\partial y^{2}}+\frac{\partial^{2} T_{p}}{\partial z^{2}}\right]=0,
$$

Where, $T$ is the temperature and $x, y, z$ are the spatial coordinates. The subscript $p$ here refers the base polymer-matrix. The parameter such as $K$ refers the thermal conductivity, pis the density, and $c_{p}$ is the specific heat. These parameters better reflect the local properties of the 3-D grid cell. In our numerical simulations, the thermal conductivity of the matrix-polymer $K_{p}$ is set to $0.29 \mathrm{~W} / \mathrm{m}-\mathrm{K}$, which corresponds to the thermal conductivity of the polyethylene.

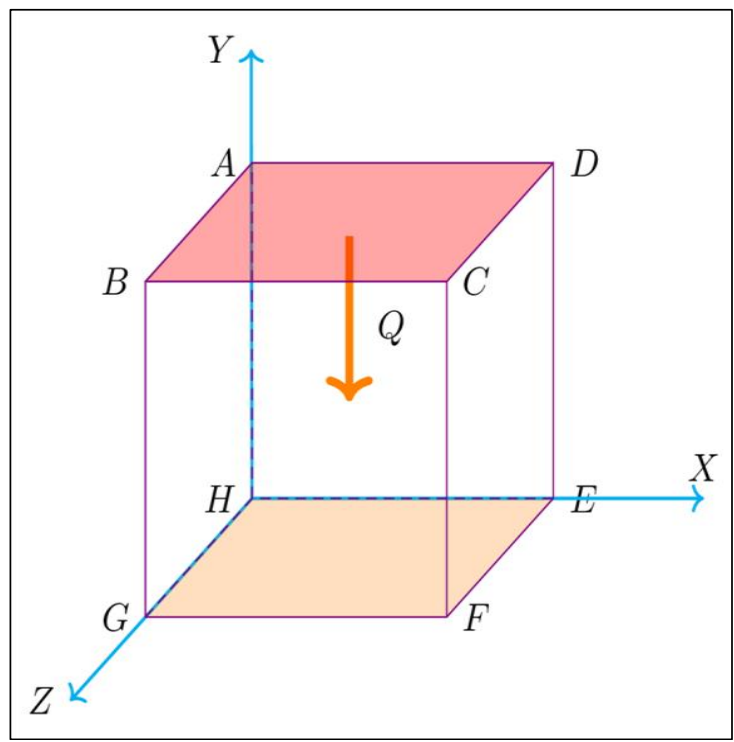

Fig. 1: A Schematic Diagram Showing the Polymer-Composite in the Shape of Cubical

Box. Note that the Empty Volume is filled with

Matrix of Polymer. The Top and Bottom

Surfaces are shown with Two Different

Colours. The Direction of Heat-Flux is from

Top to the Bottom Surface in the Negative Y-

Direction. Top Surface is kept at a Constant Temperature.
The heat transfer equation corresponding to the high conducting filler material is similar to the Eq. (1) and is given by:

$$
\frac{K_{f}}{\left(\rho c_{p}\right)_{f}}\left[\frac{\partial^{2} T_{f}}{\partial x^{2}}+\frac{\partial^{2} T_{f}}{\partial y^{2}}+\frac{\partial^{2} T_{f}}{\partial z^{2}}\right]=0
$$

Here the subscript $f$ refers to the filler. We note that the filler material here taken is Aluminium, and the corresponding thermal conductivity $K_{f}$ which is set to be $205.0 \mathrm{~W} / \mathrm{m}$ $\mathrm{K}$. Note that aluminium is very soft and nonmagnetic metal. It can be given any shape due to its soft and ductile nature. Also, aluminium is used as a good heat-exchanger in many devices due to its high conductivity and lowcost alternative. Although copper is the excellent thermal conductor $(385.0 \mathrm{~W} / \mathrm{m}-\mathrm{K})$ but it is not cost-effective and hence not widely used on the large scale. Other metals which can be used as filler materials are brass $(109.0 \mathrm{~W} / \mathrm{m}-\mathrm{K})$, iron $(79.50 \mathrm{~W} / \mathrm{m}-\mathrm{K})$, and steel $(50.20 \mathrm{~W} / \mathrm{m}-\mathrm{K})$ but their thermal conductivity is very low.

Now we write the boundary condition at the top surface $A B C D$ of the cell which is given by:

$$
-\left.K_{p} \frac{\partial T}{\partial n}\right|_{\Gamma}=Q
$$

Where, $Q$ is the heat flux which is kept steady and constant on the top surface ABCD, and $n$ is the normal to the surface towards the downwards low temperature surface in the direction of heat flux. Note that the symbol Trepresents the planar surface spanned by EFGH. This is known as the secondclass boundary condition. In our numerical calculations, we set $Q$ to be $1 \mathrm{~kW} / \mathrm{m}^{2}$. Another class of boundary condition which is known as third class boundary condition is set on the bottom surface EFGH of the 3-dimensional cell which is:

$$
\left.K_{p} \frac{\partial T}{\partial n}\right|_{\Gamma}=\left.h\left(T-T_{f}\right)\right|_{\Gamma}
$$

Here the convective heat transfer coefficient $h$, and the ambient extensive temperature $T_{f}$ are set to be constants. In our numerical simulations, we have set $h$ to be $20 \mathrm{~W} / \mathrm{m}^{2} \mathrm{~K}$, and $T_{f}$ is set to be $300 \mathrm{~K}$. Apart from these two specific boundary conditions (i.e., for top and bottom surfaces), we have four more boundary conditions for the remaining planes corresponding to each of the following 
surfaces EHAD, HGBA, FGBC, and EFCD, which can be written as:

$$
\left.\frac{\partial T}{\partial n}\right|_{\Gamma}=0
$$

Here, $\Gamma$ represents the four surfaces mentioned above. These boundary conditions clearly mention that the surfaces are adiabatic and there is no heat flux through these surfaces.

\section{Heat Transfer Coupling}

Note that at the interface of the polymer and filler material, i.e., at the contact surface area, the heat-flux is same. The flow of heat in polymer and filler are thus coupled. This coupling is very important to determine the effective conductivity of the composite polymer-filler material. This can be written explicitly as follows:

$$
-\left.K_{p} \frac{\partial T_{p}}{\partial n}\right|_{\Gamma}=-\left.K_{f} \frac{\partial T_{f}}{\partial n}\right|_{\Gamma}
$$

Where, subscript represents the contact surface amid the base matrix and the fillers. When the temperature fields in the cell are calculated, the effective thermal conductivity in $y$ direction for the cell is estimated by the following equation (Figure 1):

$$
K_{c}=\frac{l_{y}}{\left(\Delta T_{\text {cell }}\right)} Q
$$

Where, $\Delta T$ is the mean temperature difference between the top and bottom surface of the cell. The surface means temperature here is bounded by area $l_{x}-l_{y}$. Dimension along the $x$ axis is $l_{x}$, dimension along the $\mathrm{y}$ axis is $l_{y}$ and dimension along $\mathrm{z}$ axis is $l_{z}$ and they are the cell length, height and width respectively. We also write the thermal conductivity explicitly as:

$$
\begin{aligned}
& Q=-K A \frac{(\Delta T)}{(\Delta X)} \\
& K=-\frac{Q}{A} \frac{(\Delta X)}{(\Delta T)}
\end{aligned}
$$

Where, $K$ is thermal conductivity (W/m-K), and $Q$ is the total heat transfer (variable). $\Delta T$ is the difference in temperature $T_{1}-T_{2} . T_{1}$ is fixed at $400 \mathrm{~K}$, and $\mathrm{T}_{2}$ is variable. $\mathrm{A}$ is the area which is given by $0.01 \times 0.01 \mathrm{~m}^{2} . \Delta X$ is thickness through which heat conduction takes place. Here, $K=K_{c}$ (thermal conductivity of composites material, and $\mathrm{K}_{\mathrm{c}} / \mathrm{K}_{\mathrm{p}}$ is the relative thermal conductivity.

\section{Simulation Details}

Three dimensional aluminium fillers have been designed and inserted in the matrix polymer to generate the composite polymers. The fillers of variable shape and size have been used which depend on the volume percent of the filler. The details of shape and size have been described in the result and discussion. We have used Ansys-18.0 for our numerical simulations, which is based on the finite element method. The simulation box (i.e., the outer shape of the composite polymer) is chosen as a cubical box. The dimension of the box is $0.01 \times 0.01 \times 0.01 \mathrm{~m}^{3}$. The volume of the box is sufficient for 3-D simulations. Note that the height of the filler is kept maximum to facilitate the longer path for the heat-flux, which is very effective in the maximization of the effective thermal conductivity. Our main objective is to design the fillers of best shape such that they can maximize the effective thermal conductivity. In this regard we have fabricated fillers of various shapes and sizes. We generally start with the known geometries as they have well defined shape and can make the comparison between their qualitative features.

We have created all the fillers of length $0.5 \mathrm{~cm}$ except the sphere and the filler percentage is taken within the range 1-9\%. The widths of filler taken in the shape of hollow cylinders are $0.26242,0.27428,0.28565,0.29659$, $0.30713,0.31733$ and $0.34612 \mathrm{~cm}$ respectively with the corresponding thicknesses 0.01242 , $0.02428,0.03565,0.04659,0.10713,0.16633$ and $0.19512 \mathrm{~cm}$ respectively. The solid cylinder of widths $0.029735,0.05945$, $0.089205,0.11894,0.148675,0.17841$ and $0.267615 \mathrm{~cm}$ respectively. The I-shape filler of thicknesses $0.05,0.1,0.15,0.2,0.25,0.3$ and $0.35 \mathrm{~cm}$ respectively. The T-shape filler of thicknesses $0.07,0.115,0.175,0.2282,0.285$, 0.3426 and $0.4325 \mathrm{~cm}$ respectively. The Xshape filler of thicknesses 0.0342, 0.06, 0.079, $0.10,0.119,0.145$ and $0.195 \mathrm{~cm}$ respectively. The Y-shape filler of thicknesses $0.0989,0.15$, $0.224,0.2495,0.312,0.345$ and $0.448 \mathrm{~cm}$ respectively. Sphere shape filler of radius $0.135,0.169,0.195,0.212,0.2285,0.2429$ and $0.278 \mathrm{~cm}$ respectively. Here we have also compared with the correlation models with the simulation result in Figure 12. 


\section{RESULTS AND DISCUSSION}

In this section, we describe in detail the results of our numerical simulations. We have used various fillers of different shapes and sizes to measure the effective thermal conductivity.

We consider the composite polymer as twophase system (the two phases being $a$ and $b$ respectively). Phase $a$ corresponds to the bare polymer, and the other phase $b$ corresponds to the metallic filler. Let the concentration, i.e., the volume fraction of the filler is $x$, then the volume fraction of the polymer is defined to be $1-x$, such that sum of the total volume is 1 . Their ratio $x /(1-x)$ determines the nature or the behaviour of the composite polymer. If $x=0$, i.e., there is complete absence of the filler then this represents the $100 \%$ of the pure matrix polymer. On the other hand for $x=1$, it represents the complete metal. In general, the value of $x$ is taken to be very low. In our simulations, the volume fraction of the filler i.e., $x$, varies from 0 to $9 \%$ which is within the $10 \%$ of the composite material. The interface which is the boundary between the two phases or the two components is an important quantity to dictate the effective thermal conductivity of the composite. Note that in the fabrication of the composite polymer it should be ensured that the phases $a$ and $b$ are not chemically reactive.

We would like to stress the fact that the shape of the filler is designed such that it can maximize the thermal conductivity. Hence the underlying mechanism should be such that the dispersion of phase $a$ into phase $b$ can perform this task. We would also like to mention that there is no as such any fundamental mechanism which has a guideline to enhance the thermal conductivity accordingly.

However, if we try to understand the propagation of heat flux in the polymer in context of heat-flux in metals, the basic mechanism of thermal conduction in polymers can be understood up to a large extent. For example, the phonons are carriers of the heat flux of the local thermal vibrations in the metal. These phonons can propagate linearly from one point to another spanning a long distance. On the other hand, in polymers, due to their complex branching and complex molecular structure, the propagation of phonons is restricted locally making them very low thermal conductors. If we can excite the phonons to propagate for larger distances, it is possible enhance the conductivity.

In this regard, the role of individual molecules in the polymer becomes very important. Each molecule of the polymer can be treated as a unit of heat flux which has only local effect. If these molecules are excited or heated then their collective behaviour can have significant effect in the thermal conduction. This can be achieved if we can give heat to the maximum number of particles. This is possible if more molecules come into contact with the metal filler. Therefore the filler should be designed in such a way that it can transfer heat to the maximum number of polymer molecules. This is possible only when the surface area of the filler is large so that it can make contact with maximum number of molecules. Therefore, if the number of molecules participating in the thermal conduction increases, the effective role conductivity should also increase.

To outline this procedure we consider the case of a cylindrical filler. Initially we perform our simulation with a solid cylinder of a given radius $(r)$, and height $(h)$ for a given volume fraction $x$. The surface area of the cylindrical filler which is in contact with the polymer is $(2 \Pi r h)$. This is also the interface area between the two phases in the composite. We measure the effective thermal conductivity for this case.

Now we take the hollow cylindrical filler of the same outer radius $r=r_{1}$, the inner radius $r_{2}$, and height $h$. We choose the thickness of the cylinder to be very small or negligible such that there is no difference between inner and outer radius, i.e., $r_{1} \sim r_{2}=r$. In this case the total surface area of the filler material is twice compared to the previous case, i.e., when the filler is solid cylinder. Now the interface or the total contact surface area between the two phases is twice. Thus, the number of polymer molecules which come into contact with the metallic filler is just double due to this surface 
maximization. Effect of the increase in the interface (or the contact surface area) can now be clearly observed in the enhancement in effective thermal conductivity as shown in Figure 11.

This clearly indicates that interface between the two phases $a$ and $b$ plays significant role in the enhancement of the thermal conduction in composite polymers. We can propose that effective thermal conductivity is proportional to some function of contact surface area between the two phases in the composite. Thus, the effective thermal conductivity $K$ is proportional to $f(A)$, where $f(A)$ is some function of contact surface area $A$ at the interface.

Note that during maximizing the surface area, our basic assumption is that we keep the volume fraction of the filler content constant so that we can obtain the maximum interface. We do not increase or decrease the volume fraction of the filler during the surface modification.

The bulk or the volume of the filler is another important quantity which controls the thermal conductivity, however we want to keep volume fraction of the filler to be low and constant. By increasing the bulk of the filler we will definitely have the high thermal conductivity, but that will be signature of the thermal conductivity of the metal, not the polymer's thermal conductivity.

To outline our methodology we have dealt with cases of different fillers of various shapes and sizes. Figures 4-10, 13 and 15 show corresponding contour plots, representing the temperature gradients in different parts of the composite polymer in the steady state. In Figure 11 we compare the effective thermal conductivity with varying volume fraction $x$ in all the cases. Note that surface maximization of the filler has no definite rule, and the geometry for a given volume fraction can have variable surface area depending on the thickness. However, we try to get the maximum surface area by making the surface thinner and thinner giving it to a microscopic thickness without any loss in the basic property of the filler.
Now we propose the basic question again that what are the best shape fillers to enhance the effective thermal conductivity in composite polymers. Our numerical simulations suggest that we can enhance the thermal conductivity by surface maximization of the filler keeping the volume percent constant without increasing the filler's volume fraction. To give more strong support in this context, we choose the different geometries for which the surface $(S)$ to the volume $V$ ratio $S / V$ is very high. We have chosen three such geometries which have high surface to volume ratio and their order for $S / V$ is octahedron < cube < tetrahedron. Their contour plots have been shown in the Figures 13-15 respectively. In Table 2 we have tabulated their description and the values of effective thermal conductivities for the comparison. It is clear from the table that the effective thermal conductivity in these cases is also in the same order as that of $S / V$ ratio, validating our claim.

Therefore, it is clear that role of interface, i.e., the contact surface area plays the major role in enhancing the effective thermal conductivity in polymer composites. This is achieved by the surface maximization of filler material.

Now we give the detailed descriptions of the figures corresponding to the different cases considered in our simulations. In Figure 2 we show the mesh structure of a composite polymer filled with metallic filler. The shape of filler is cylindrical which is hollow, the top surface $A B C D$ is at a constant high temperature, and the bottom surface is $E F G H$ where the effect of heat flux is noted. Heat flux is from top to the bottom surface. In the whole empty volume of the structure, the base polymer is filled to avoid any void. The inside bulk of hollow cylinder is also replaced by the base polymer. Therefore, the polymer is filled within and outside the cylindrical filler. Note that contact area between polymer filler has now been two-fold increased. The volume percent of filler in this case is only $9 \%$.

In Figure 3 we show the three-dimensional contour representing the temperature gradient from top to the bottom surface in the static 


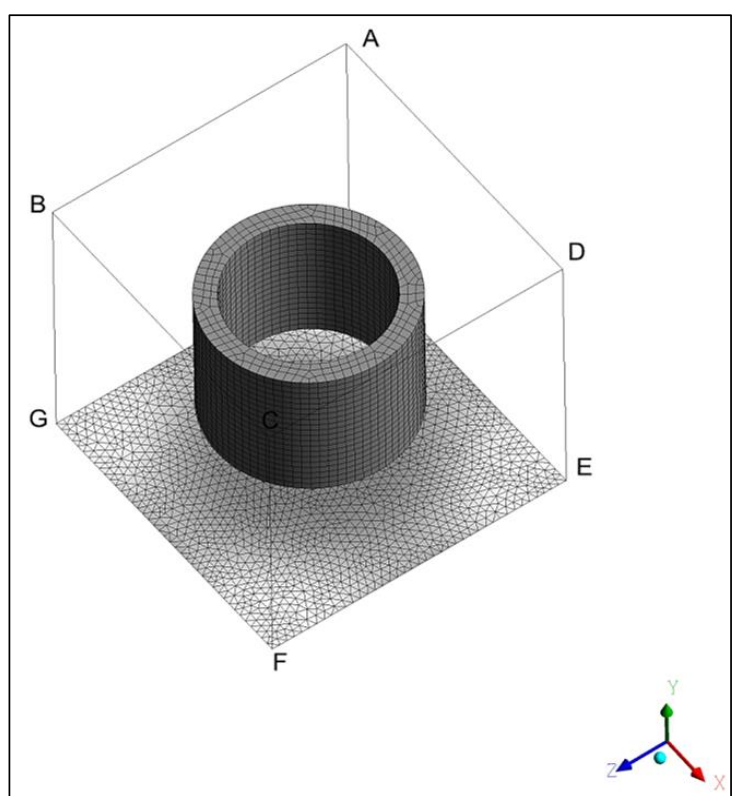

Fig. 2: The Mesh Structure of a Composite Polymer which has a Metallic Filler in the Shape of Hollow Cylinder. In the Whole Empty Volume the Base Polymer is Filled Again. Therefore Polymer is Filled within and Outside the Cylindrical Area. Note that Contact Area between Polymer and Filler has been Two-Fold Increased in This Case Also.

The Bulk Inside is Replaced by the Base

Polymer. The Volume Percent of Filler in this Case is Only 9\%.

condition for a filler in the shape of hollow cylinder corresponding to Figure 2. The top surface represents the maximum temperature kept constant at $400 \mathrm{~K}$ which is uniform across the surface. The volume percent of filler in this case is only $9 \%$.

In Figure 4 we show the two-dimensional contour in the composite polymer representing the heat flux from the top to the bottom surface. At the top surface temperature is kept constant at $400 \mathrm{~K}$, and at the bottom temperature in the steady state is $365.44 \mathrm{~K}$ after simulation. The horizontal lines show the variations of temperature across the surface at different vertical lengths. The figure corresponds to the solid cylindrical filler with filler content of $9 \%$ and of filler length $0.5 \mathrm{~cm}$.

In Figure 5 we show the two-dimensional contours of temperature gradient for the crosssection in the $x-z$ plane $\left(\right.$ at $\left.y=0.51_{y}\right)$ for the composite polymer. This is representing the heat flux from the top to the bottom surface. At the top surface, temperature is kept constant at $400 \mathrm{~K}$, and at the bottom, temperature is $368.14 \mathrm{~K}$ after simulation. The horizontal lines show the variations of temperature across the surface at different vertical lengths. The figure corresponds to the hollow cylindrical filler with volume fraction of $9 \%$, and of the filler length $0.5 \mathrm{~cm}$.

In Figure 6 we show the two dimensional contour of temperature gradient for the crosssection in the $x-y$ plane (at $\mathrm{z}=0.5 \mathrm{l}_{\mathrm{z}}$ ) for the composite polymer representing the heat flux from the top to the bottom surface. At the top surface, temperature is kept constant at $400 \mathrm{~K}$, and at the bottom, temperature is $367.83 \mathrm{~K}$ after the simulation. The horizontal lines show the variations of temperature across the surface at different vertical lengths. The figure corresponds to the I-shape filler with filler fraction of $9 \%$, and of the filler length $0.5 \mathrm{~cm}$.

Figure 7 corresponds to the two dimensional contour of temperature gradient for the crosssection in the $z-y$ plane (at $\mathrm{x}=0.51_{\mathrm{x}}$ ) for the composite polymer representing the heat flux from the top to the bottom surface. At the top surface, temperature is kept constant at $400 \mathrm{~K}$, and at the bottom surface, temperature in the steady state is $367.23 \mathrm{~K}$ after simulation. The horizontal lines show the variations of temperature across the surface at different vertical lengths. The figure corresponds to the $X$-shape filler with the filler fraction of $5 \%$, and of filler length $0.5 \mathrm{~cm}$.

Figure 8 corresponds to the two dimensional contour of the temperature gradient for the cross-section in the $z-x$ plane (at $\mathrm{y}=0.51_{\mathrm{y}}$ ) for the composite polymer representing the heat flux from the top to the bottom surface. At the top surface, the temperature is kept constant at $400 \mathrm{~K}$, and at the bottom surface, temperature in the steady state is $366.49 \mathrm{~K}$ after the simulation. The horizontal lines show the variations of temperature across the surface at different vertical lengths. The figure corresponds to the $Y$-shape filler with filler content of $9 \%$, and of the filler length $0.5 \mathrm{~cm}$. 


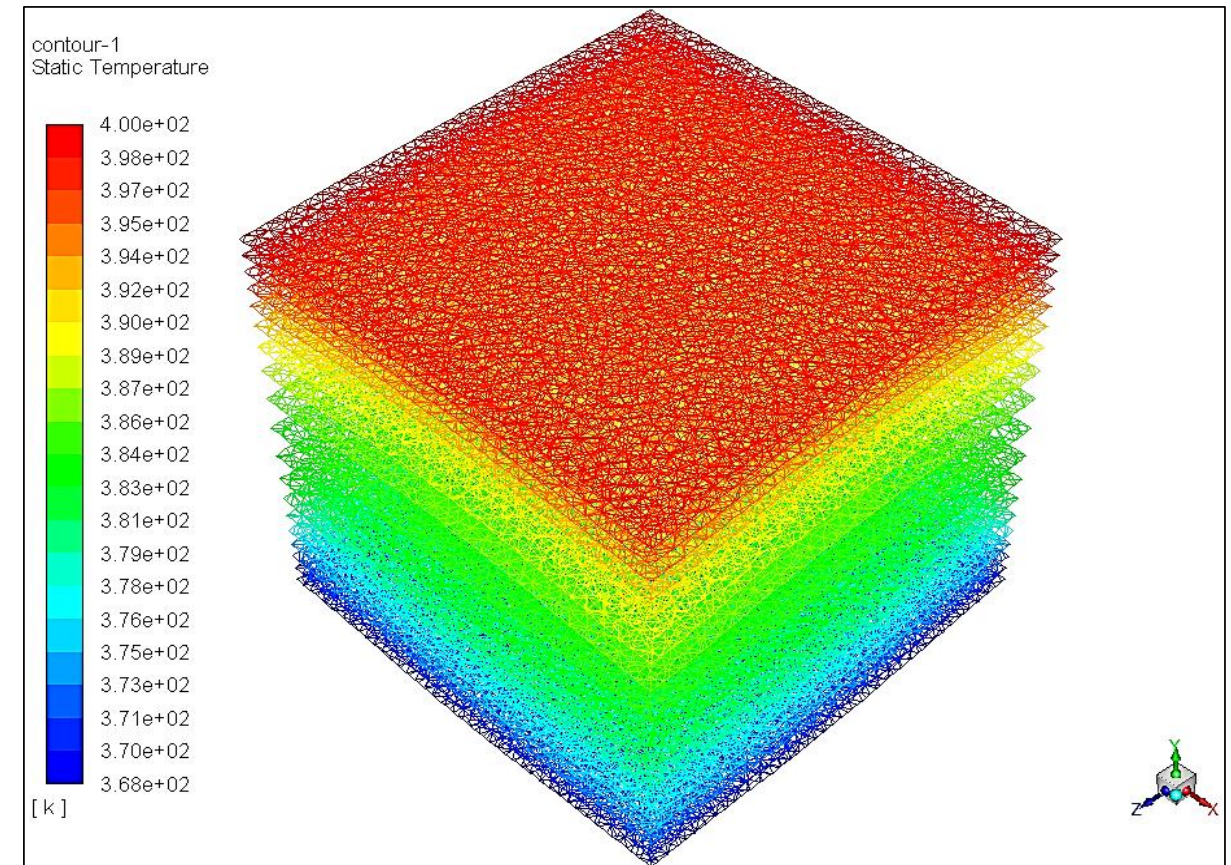

Fig. 3: The Three Dimensional Contour Representing the Temperature Gradient from Top to the Bottom Surface in the Static Condition for a Filler in the Shape of Hollow Cylinder. The Top Surface Represents the Maximum Temperature Kept Constant at $400 \mathrm{~K}$ which is Uniform across the Surface. The Volume Percent of Filler in this Case is Only 9\%.

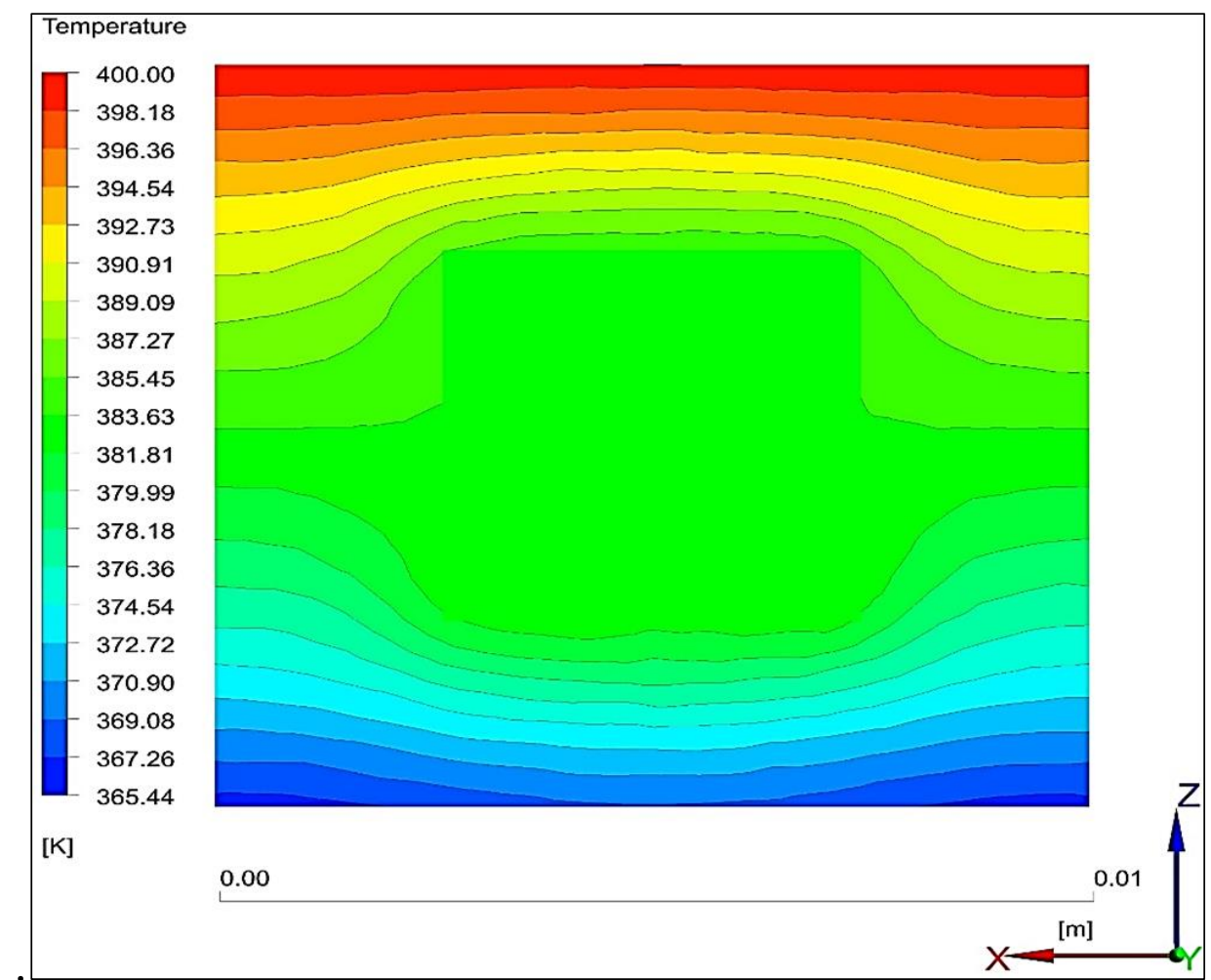

Fig. 4: The Two Dimensional Contour of Temperature $(K)$ of the Cross-Section $x$ - $z$ Plane $\left(y=0.5 l_{y}\right)$ for the Composites Polymer Representing the Heat Flux from the Top to the Bottom Surface. The Horizontal Lines Show the Variations of Temperature across the Surface at Different Vertical Length. The Figure Corresponds to the Solid Cylinder Filler with Filler Content of 9\%, and of Filler Length $0.5 \mathrm{~cm}$. 


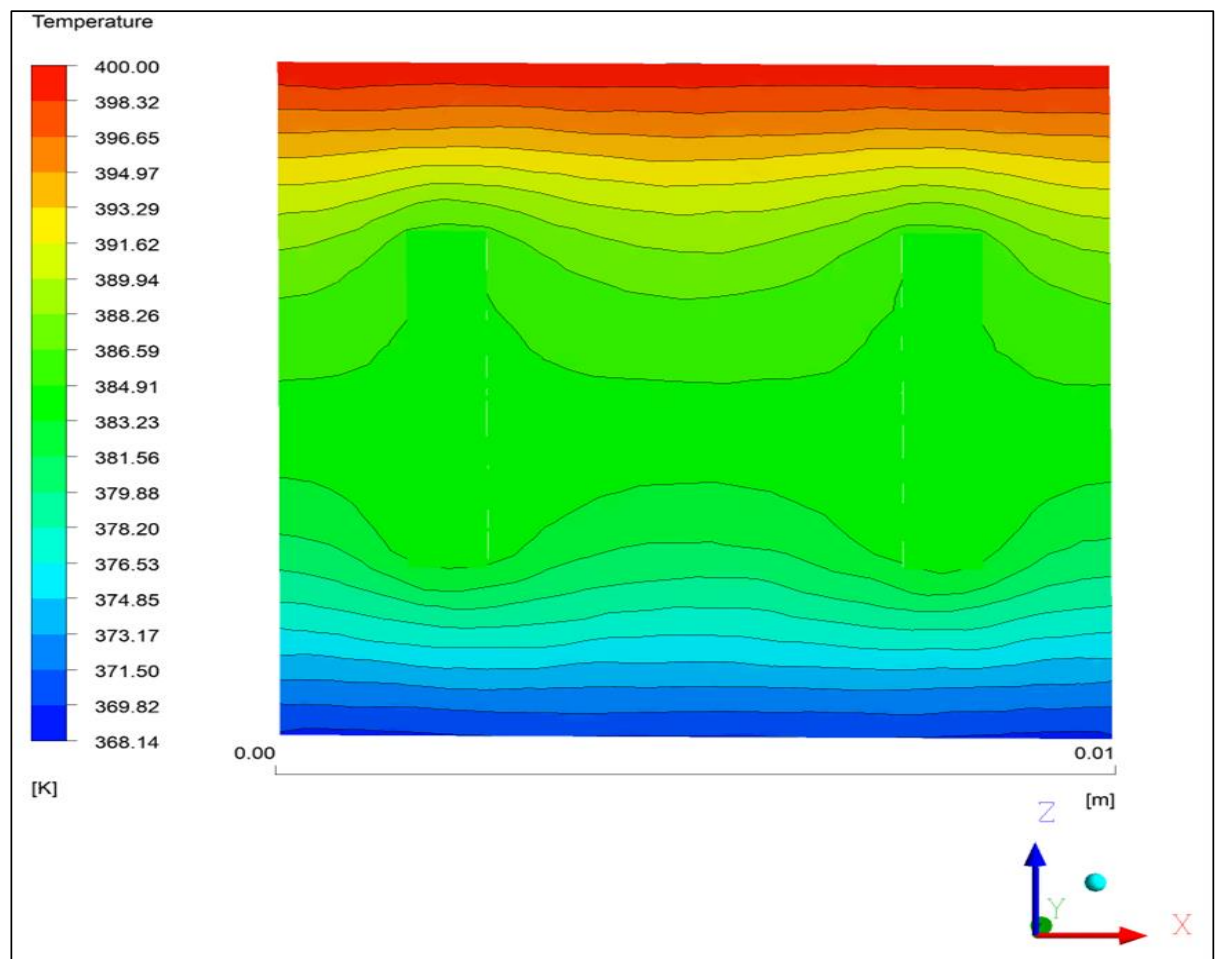

Fig. 5: The Two Dimensional Contours of Temperature $(K)$ of the Cross-Section $x$ - $z$ Plane $\left(y=0.5 l_{y}\right)$ for the Composite Polymer Representing the Heat Flux from the Top to the Bottom Surface. The Horizontal Lines Show the Variations of Temperature across the Surface at Different Vertical Length. The Figure Corresponds to the Hollow Cylinder Filler with Filler Content of 9\%, and of Filler Length $0.5 \mathrm{~cm}$.

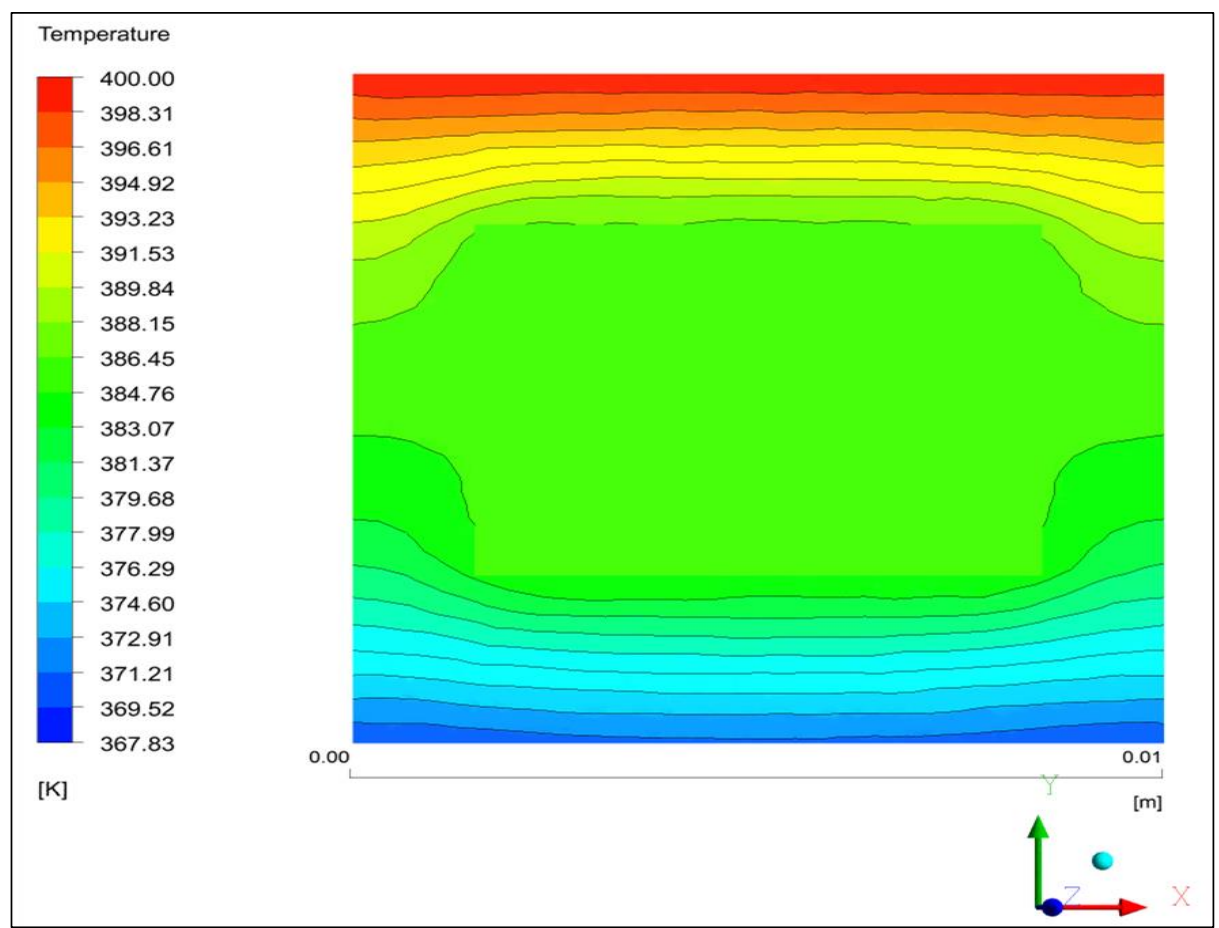

Fig. 6: The Two Dimensional Contour of Temperature $(K)$ of the Cross-Section $x$-y Plane $\left(z=0.5 l_{z}\right)$ for the Composite Polymer Representing the Heat Flux from the Top to the Bottom Surface. The Horizontal Lines Show the Variations of Temperature across the Surface at Different Vertical Length. The Figure Corresponds to the I-Shape Filler with Filler Content of 9\%, and of Filler Length $0.5 \mathrm{~cm}$. 


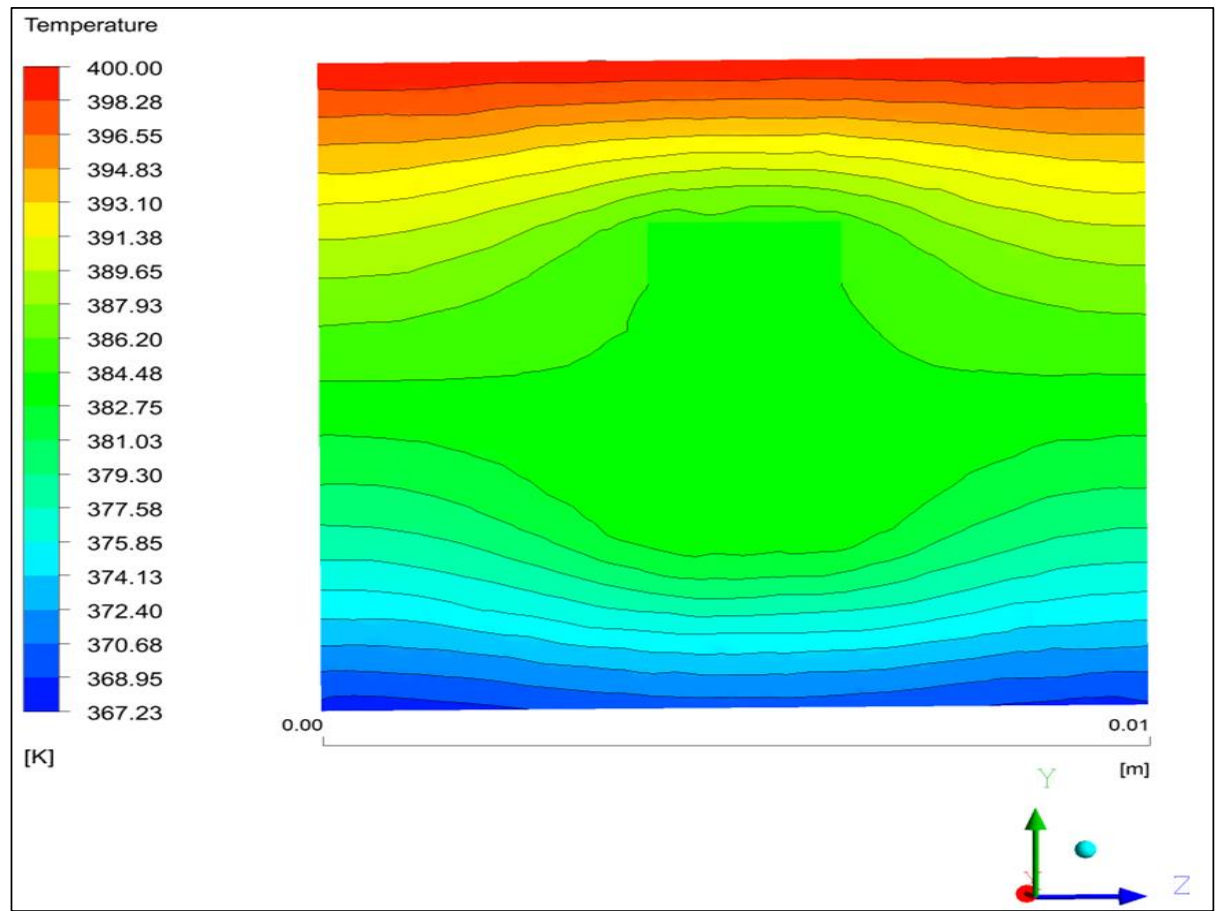

Fig. 7: The Two Dimensional Contour of Temperature $(K)$ of the Cross-Section $z-y$ Plane $\left(x=0.5 l_{x}\right)$ for the Composite Polymer Representing the Heat Flux from the Top to the Bottom Surface. The Horizontal Lines Show the Variations of Temperature across the Surface at Different Vertical Length. The Figure Corresponds to the X-Shape Filler with Filler Content of 5\%, and of Filler Length $0.5 \mathrm{~cm}$.

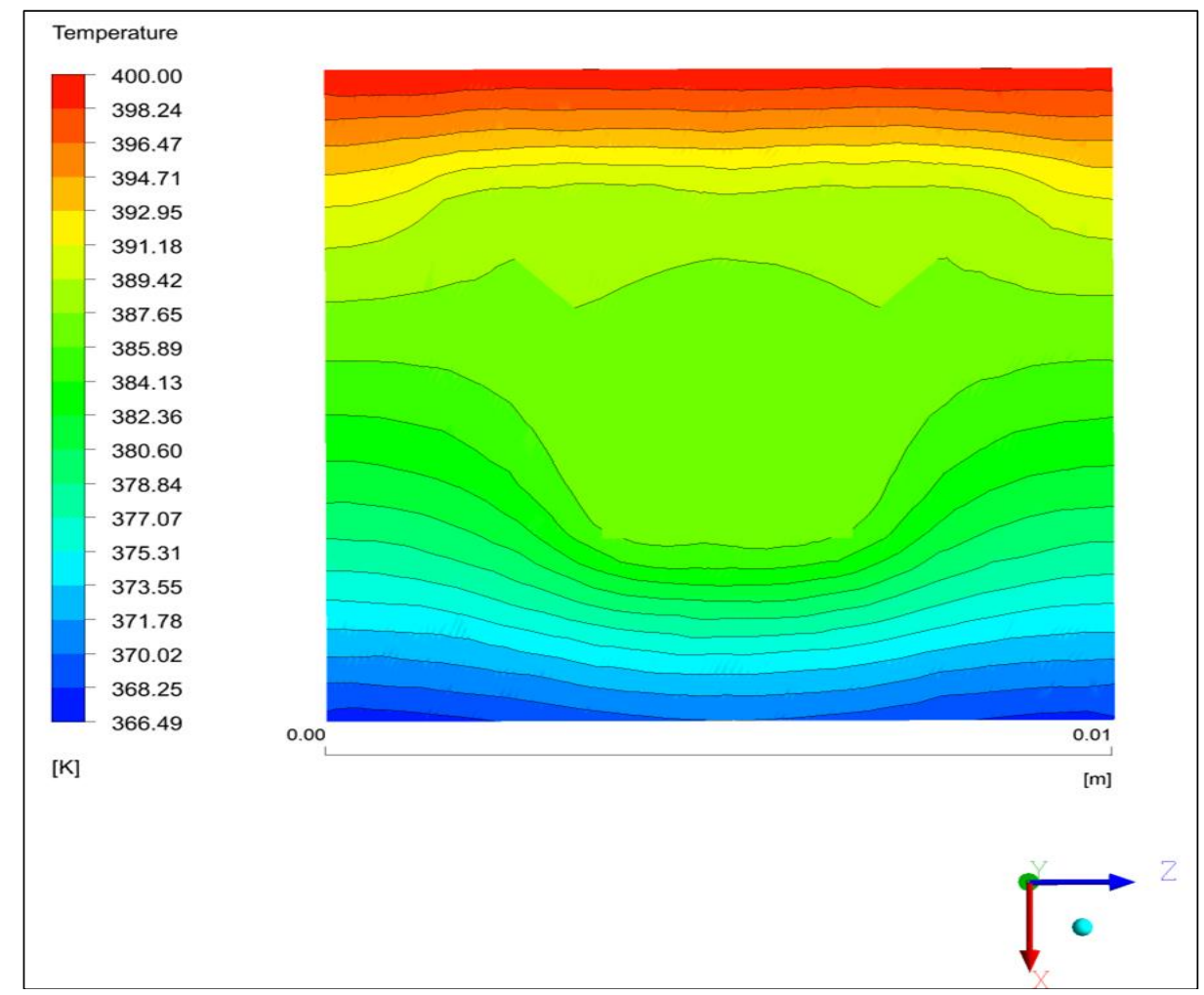

Fig. 8: The Two Dimensional Contour of Temperature $(K)$ of the Cross-Section z-x Plane $\left(y=0.5 l_{y}\right)$ for the Composite Polymer Representing the Heat Flux from the Top to the Bottom Surface. The Horizontal Lines Show the Variations of Temperature across the Surface at Different Vertical Length. The Figure Corresponds to the Y-Shape Filler with Filler Content of 9\%, and of Filler Length $0.5 \mathrm{~cm}$. 
In Figure 9 we show the two dimensional contours of temperature gradient for the crosssection $x$ - $y$ plane (at $\mathrm{z}=0.5 \mathrm{l}_{\mathrm{z}}$ ) for the composite polymer representing the heat flux from the top to the bottom surface. At the top surface, temperature is kept constant at $400 \mathrm{~K}$, and at the bottom surface, temperature in the steady state is $366.39 \mathrm{~K}$ after the simulation. The horizontal lines show the variations of temperature across the surface at different vertical lengths. The figure corresponds to the $T$-shape filler with filler content of $9 \%$, and of filler length $0.5 \mathrm{~cm}$.

Figure 10 corresponds to the two dimensional contour of temperature gradient for the crosssection in the $z-y$ plane $\left(\right.$ at $\left.\mathrm{x}=0.51_{\mathrm{x}}\right)$ for the composite polymer representing the heat flux from the top to the bottom surface. At the top surface, temperature is kept constant at $400 \mathrm{~K}$, and at the bottom surface, temperature in the steady state is $363.99 \mathrm{~K}$ after simulation. The horizontal lines show the variations of temperature across the surface at different vertical lengths. The figure corresponds to the sphere-shape filler with filler content of $9 \%$, and of radius $0.278 \mathrm{~cm}$.

In Figure 11 we see the effective thermal conductivity by increasing the volume fraction of filler; in this figure, HCYL means hollow cylinder, SCYL means solid cylinder, ISHP means I-shape, XSHP means X-shape, YSHP means Y-shape, TSHP means T-shape and SSHP means sphere shape.

Figure 12 shows that comparison of correlations model like Maxwell, Russell and Baschirow-Selenew with hollow cylinder filler. Simulation result of hollow cylinder filler is near about Russell model [17-19].

Figure 13 corresponds to the octahedron-shape filler, and shows the two dimensional contour of temperature gradient for the cross-section in the $x-y$ plane (at $\mathrm{z}=0.5 \mathrm{l}_{\mathrm{z}}$ ) for the composite polymer representing the heat flux from the top to the bottom surface. At the top surface, temperature is kept constant at $400 \mathrm{~K}$, and at the bottom surface, temperature in the steady is $364.20 \mathrm{~K}$ after the simulation. The horizontal lines show the variations of temperature across the surface at different vertical lengths. The filler volume fraction corresponds to $9 \%$, and side length is $0.576 \mathrm{~cm}$. The surface area of the octahedron filler is $1.1493 \mathrm{~cm}^{2}$.

Figure 14 corresponds to the cube-shape filler, and shows the two dimensional contour of temperature gradient for the cross-section in the $x$-z plane $\left(\right.$ at $\mathrm{y}=0.5 \mathrm{l}_{\mathrm{y}}$ ) for the composite polymer representing the heat flux from the top to the bottom surface. At the top surface, temperature is kept constant at $400 \mathrm{~K}$, and at the bottom surface, temperature in the steady state is $364.43 \mathrm{~K}$ after the simulation. The horizontal lines show the variations of temperature across the surface at different vertical lengths. The filler volume fraction corresponds to $9 \%$, and side length $0.448 \mathrm{~cm}$. The surface area of cubic filler is $1.204724 \mathrm{~cm}^{2}$.

Figure 15 corresponds to the tetrahedron-shape filler, and shows the two dimensional contour of temperature gradient for the cross-section in the $z-y$ plane $\left(\right.$ at $\left.\mathrm{x}=0.51_{\mathrm{x}}\right)$ for the composite polymer representing the heat flux from the top to the bottom surface. At the top, maximum temperature is kept constant at $400 \mathrm{~K}$ and at the bottom surface, temperature in the steady state is $365.73 \mathrm{~K}$ after the simulation. The horizontal lines show the variations of temperature across the surface at different vertical lengths. The filler content is $9 \%$, and side length is $0.915 \mathrm{~cm}$. The surface area of tetrahedron filler is 1.4501 $\mathrm{cm}^{2}$.

Note that, from all the filler contours and Tables 1 and 2, we see that the average temperature in $2 \mathrm{D}$ contour plots is different from the resultant temperature.

Table 1 shows the filler percentage, filler shape, temperature $(\mathrm{K})$, heat flux $(\mathrm{Q})$, thermal conductivity $\mathrm{K}_{\mathrm{c}}$, and relative thermal conductivity $K_{d} / K_{p}$ for fillers of different shapes. From the table it can be observed that the hollow cylinder has highest thermal conductivity among all the other fillers.

In Table 3 we show the different correlation models and the corresponding equations for the determination of effective thermal conductivity [20-27]. The different correlation models used are the Maxwell, Russell, and BaschirowSelenew. In all these cases, the particle filler fraction/content is low and has value less than $10 \%$, i.e., $\mathrm{V}_{\mathrm{f}}<10 \%$. 


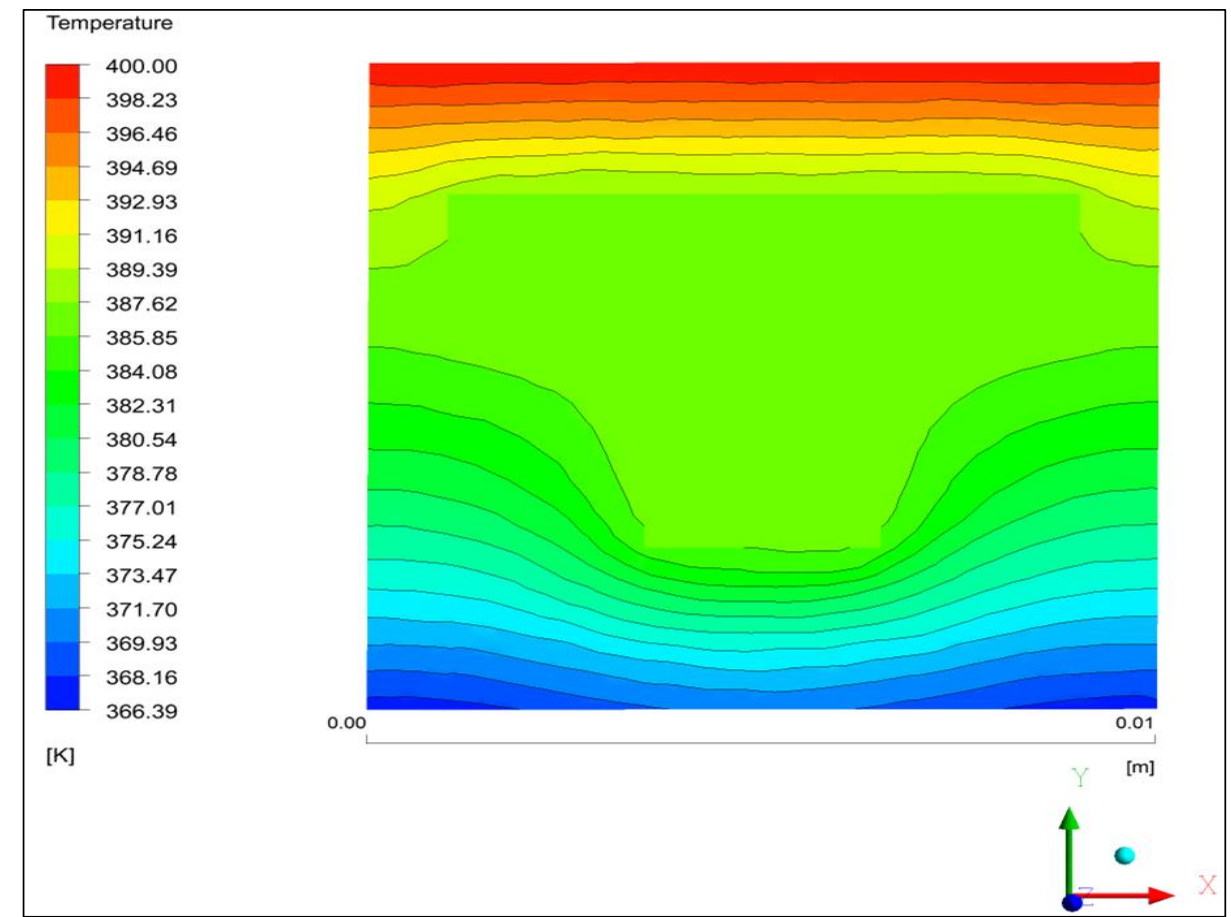

Fig. 9: The Two Dimensional Contour for of Temperature (K) of the Cross-Section $x$-y Plane $\left(z=0.5 l_{z}\right)$ the Composite Polymer Representing the Heat Flux from the Top to the Bottom Surface. The Horizontal Lines Show the Variations of Temperature across the Surface at Different Vertical Length. The Figure Corresponds to the T-Shape Filler with Filler Content of 9\%, and of Filler Length $0.5 \mathrm{~cm}$.

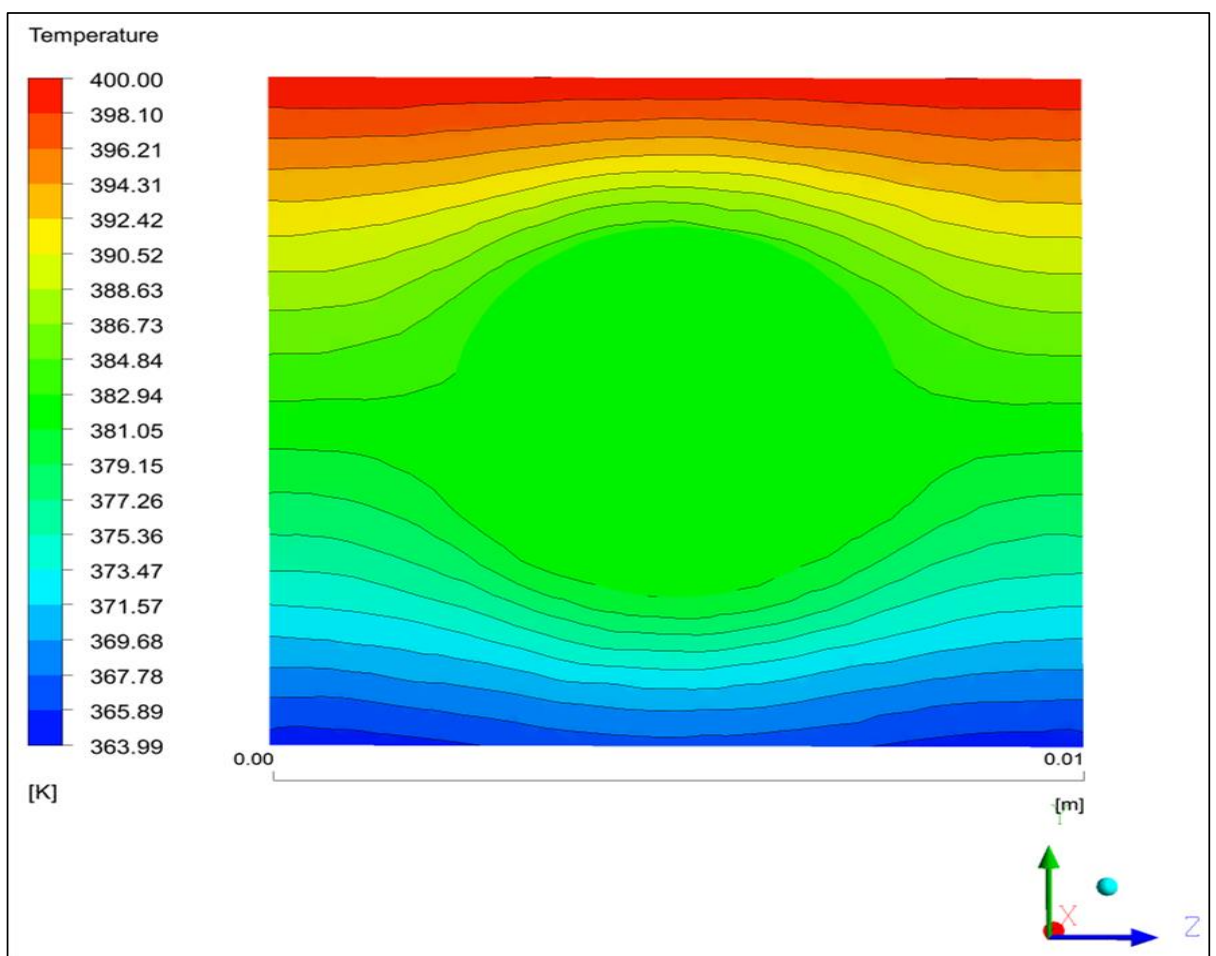

Fig. 10: The Two Dimensional Contour for of Temperature $(K)$ of the Cross-Section z-y Plane $\left(x=0.5 l_{x}\right)$ the Composite Polymer Representing the Heat Flux from the Top to the Bottom Surface. The Horizontal Lines Show the Variations of Temperature across the Surface at Different Vertical Length.

The Figure Corresponds to the Sphere-Shape Filler with Filler Content of 9\%, and of Radius $0.278 \mathrm{~cm}$. 


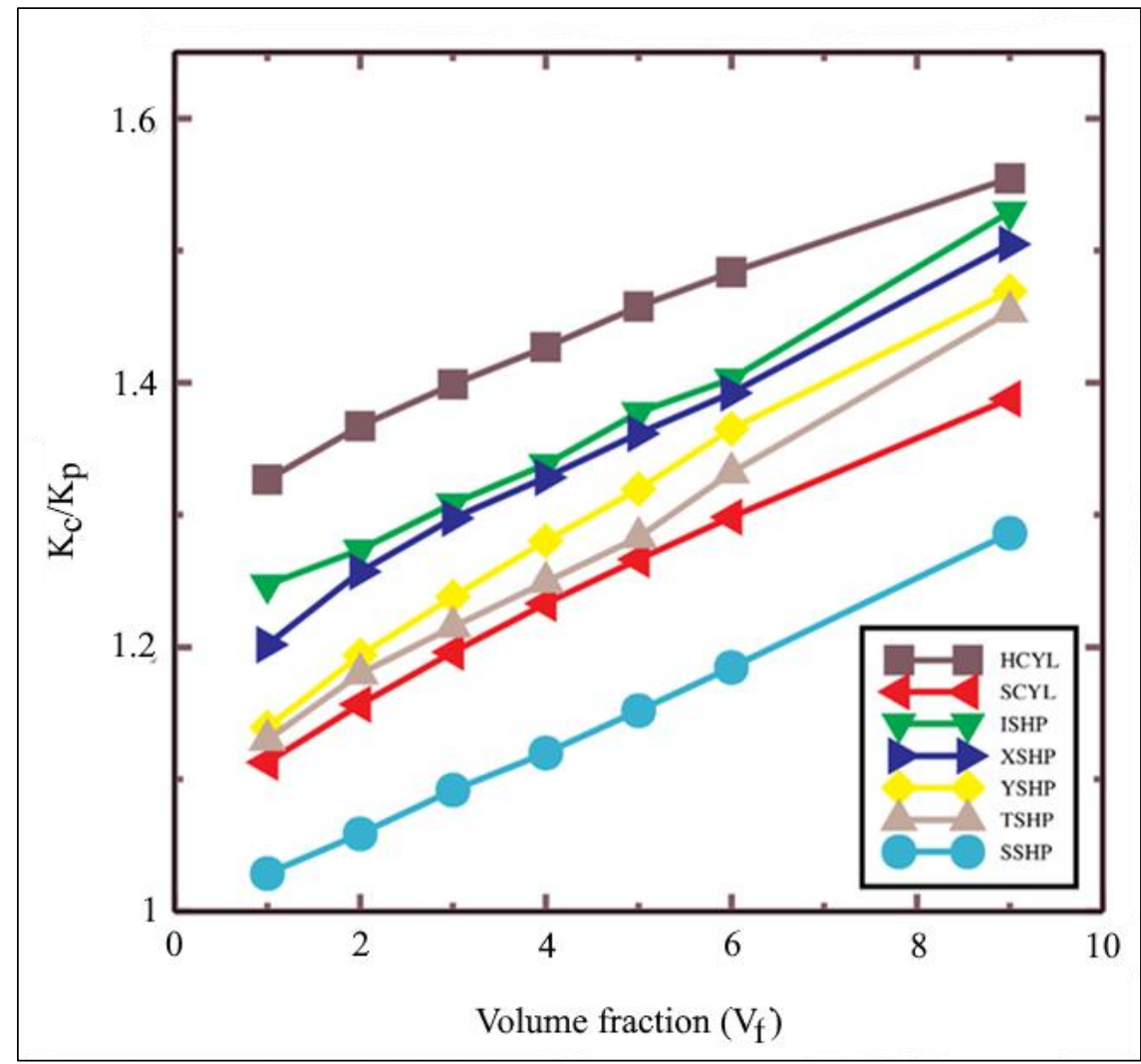

Fig. 11: Effect on Relative Thermal Conductivity by Increasing the Volume Fraction of Filler with Hollow Cylinder (HCYL), I-Shape (ISHP), X-Shape (XSHP), Y-Shape (YSHP), T-Shape (TSHP), Solid Cylinder (SCYL), and Sphere-Shape (SSHP) of Filler Length $0.5 \mathrm{~cm}$ except Sphere Shape.

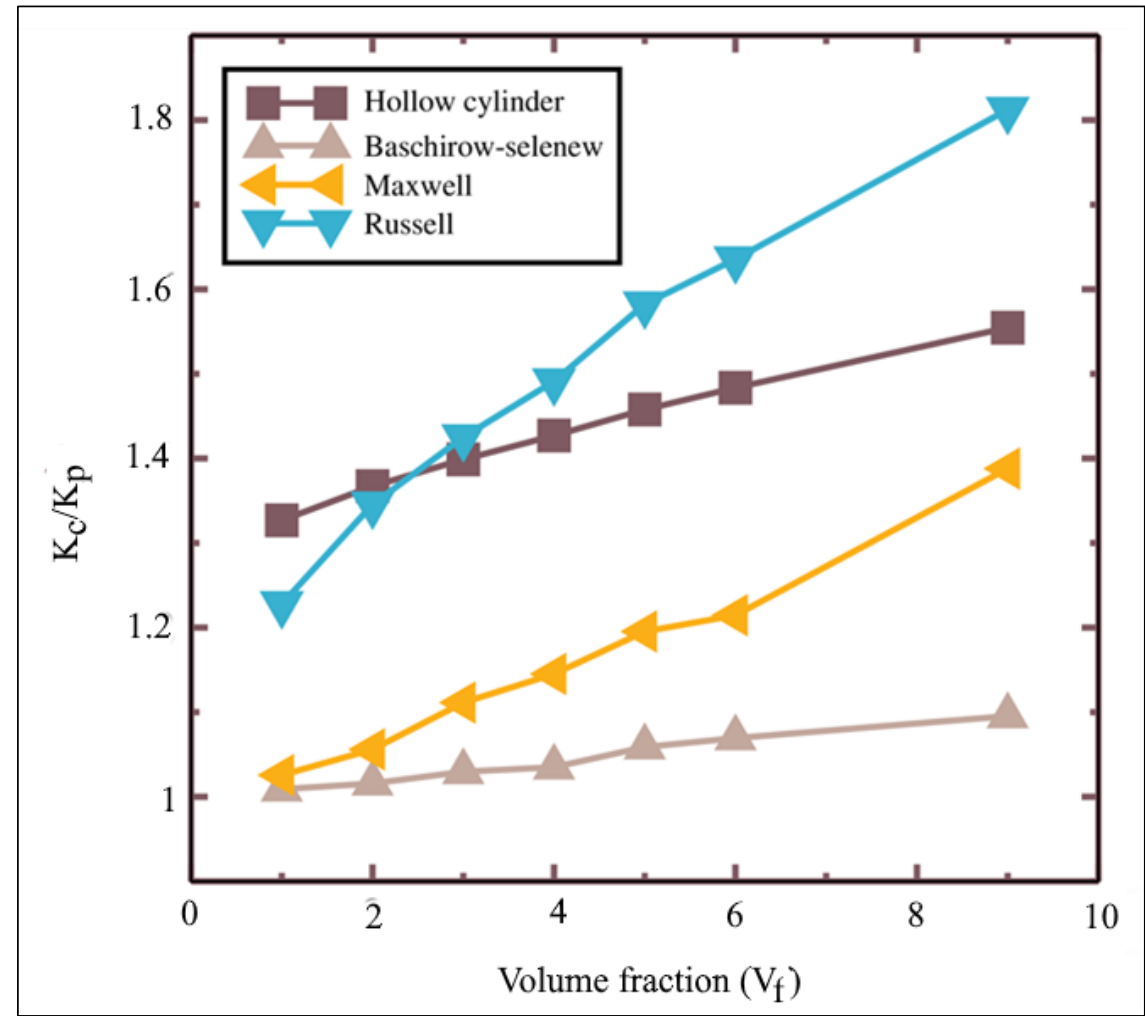

Fig. 12: Comparison of the Relative Thermal Conductivity Estimated by Correlations and Modelled. 


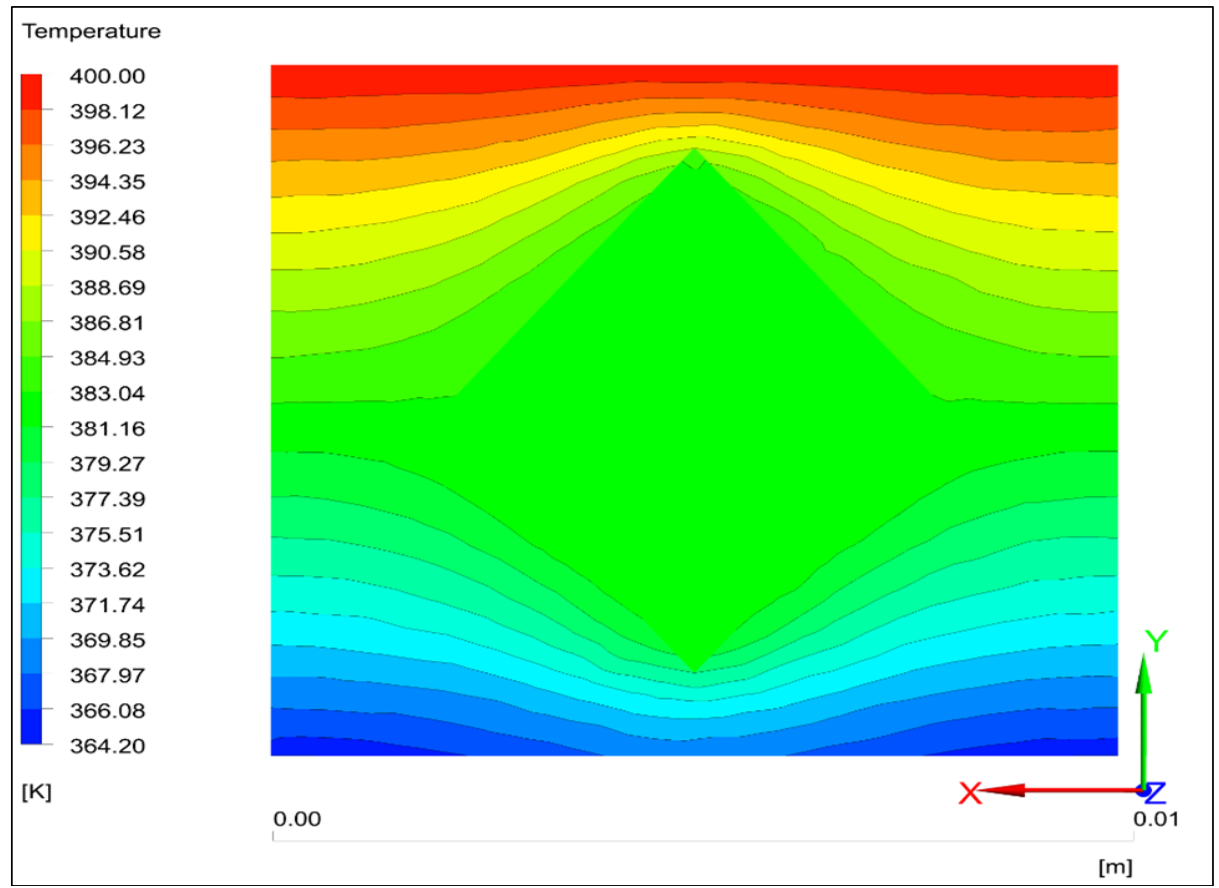

Fig. 13: The Two Dimensional Contour of Temperature $(K)$ of the Cross-Section $x$-y Plane $\left(z=0.5 l_{z}\right)$ for the Composite Polymer Representing the Heat Flux from the Top to the Bottom Surface. The Horizontal Lines Show the Variations of Temperature across the Surface at Different Vertical Length. The Figure Corresponds to the Octahedron-Shape Filler with Filler Content of 9\%, and of Side Length $0.576 \mathrm{~cm}$.

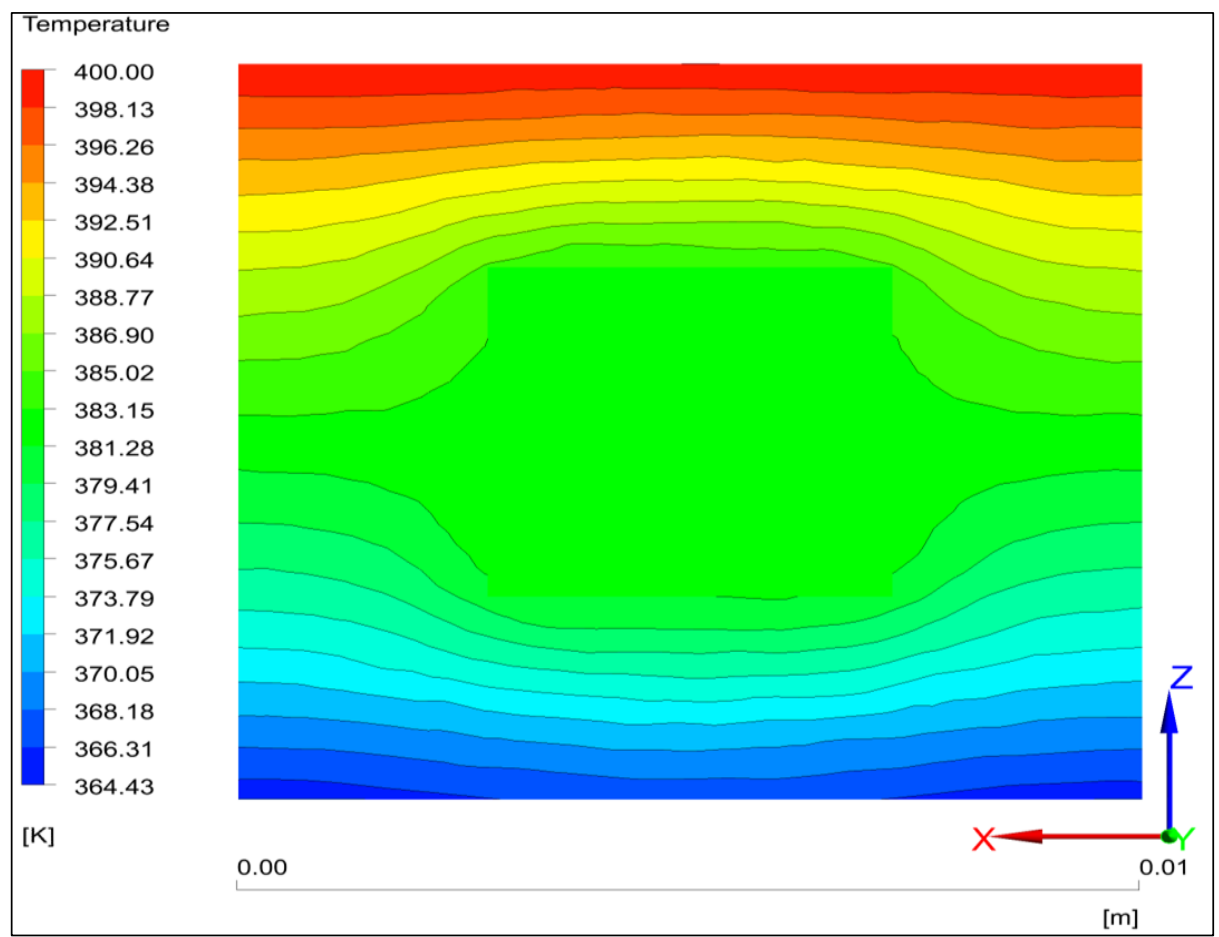

Fig. 14: The Two Dimensional Contour of Temperature $(K)$ of the Cross-Section $x-z$ Plane $\left(y=0.5 l_{y}\right)$ for the Composite Polymer Representing the Heat Flux from the Top to the Bottom Surface. The Horizontal Lines Show the Variations of Temperature across the Surface at Different Vertical Length. The Figure Corresponds to the Cube-Shape Filler with Filler Content of 9\%, and of Side Length $0.448 \mathrm{~cm}$. 


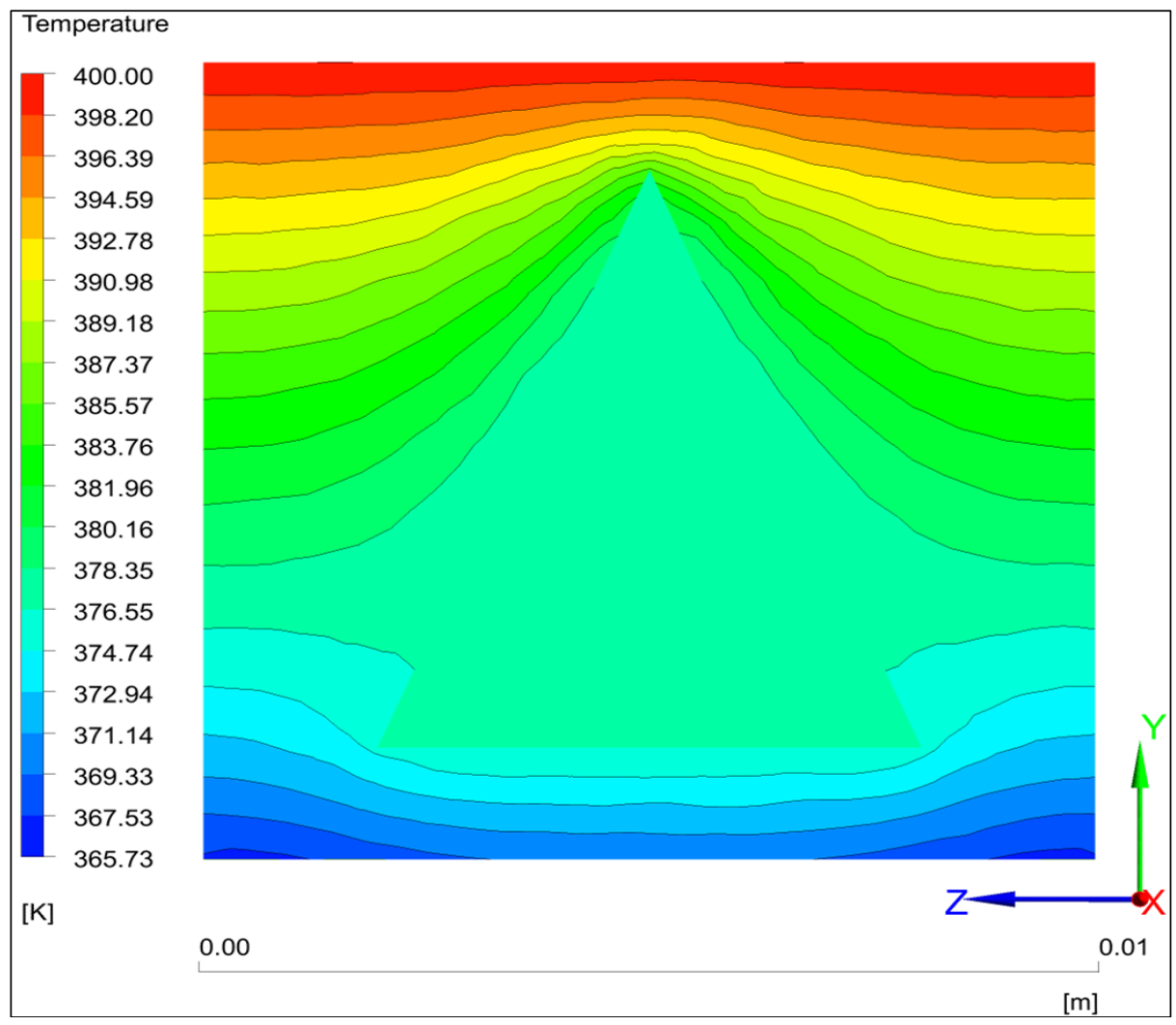

Fig. 15: The Two Dimensional Contour of Temperature $(K)$ of the Cross-Section z-y Plane $\left(x=0.5 l_{x}\right)$ for the Composite Polymer Representing the Heat Flux from the Top to the Bottom Surface. The Horizontal Lines Show the Variations of Temperature across the Surface at Different Vertical Length. The Figure Corresponds to the Tetrahedron-Shape Filler with Filler Content of 9\%, and of Side Length $0.915 \mathrm{~cm}$.

Table 1: Different Filler Shapes with the Same Filler Fraction at 9\%.

\begin{tabular}{|c|l|c|c|c|c|}
\hline $\begin{array}{c}\text { Filler } \\
\text { Percentage } \\
(\boldsymbol{\%})\end{array}$ & Filler Shape & $\begin{array}{c}\text { Temperature } \\
(\mathbf{K})\end{array}$ & $\begin{array}{c}\text { Heat } \\
\text { Flux(Q) }\end{array}$ & $\begin{array}{c}\text { Thermal } \\
\text { Conductivity } \\
\left(\mathbf{K}_{\mathbf{c}}\right)\end{array}$ & $\begin{array}{c}\text { Relative } \\
\text { Thermal } \\
\text { Conductivity } \\
\left(\mathbf{K}_{\mathbf{c}} / \mathbf{K}_{\mathbf{p}}\right)\end{array}$ \\
\hline 9 & Hollow cylinder & 369.27558425 & 0.138524808 & 0.450862301 & 1.554697592 \\
\hline 9 & I-shape & 369.23674263 & 0.136422628 & 0.443459634 & 1.529171153 \\
\hline 9 & X-shape & 368.57654866 & 0.137127535 & 0.436385979 & 1.504779239 \\
\hline 9 & Y-shape & 368.06323927 & 0.136081006 & 0.426095205 & 1.469293810 \\
\hline 9 & T-shape & 367.83190957 & 0.135620397 & 0.421599156 & 1.453790193 \\
\hline 9 & Solid cylinder & 366.81098379 & 0.133577197 & 0.402474108 & 1.387841753 \\
\hline 9 & Sphere & 365.10059790 & 0.130164012 & 0.372969176 & 1.286100609 \\
\hline
\end{tabular}

Table 2: Different Filler Shapes at Same Volume Percentage.

\begin{tabular}{|c|l|c|c|c|c|}
\hline $\begin{array}{c}\text { Filler } \\
\text { Percentage } \\
(\%)\end{array}$ & Filler Shape & $\begin{array}{c}\text { Temperature } \\
(\mathbf{K})\end{array}$ & $\begin{array}{c}\text { Heat Flux } \\
(\mathbf{Q})\end{array}$ & $\begin{array}{c}\text { Thermal } \\
\text { Conductivity } \\
\left(\mathbf{K}_{\mathbf{c}}\right)\end{array}$ & $\begin{array}{c}\text { Relative } \\
\text { Thermal } \\
\text { Conductivity } \\
\left(\mathbf{K}_{\mathbf{c}} \mathbf{\mathbf { K } _ { \mathbf { p } }}\right)\end{array}$ \\
\hline 9 & Tetrahedron & 367.69 & 0.13541 & 0.41909 & 1.44515 \\
\hline 9 & Cube & 365.45 & 0.13085 & 0.37872 & 1.30595 \\
\hline 9 & Octahedron & 365.35 & 0.13066 & 0.37708 & 1.30029 \\
\hline 9 & Sphere & 365.10 & 0.13016 & 0.37295 & 1.28610 \\
\hline
\end{tabular}


Table 3: The Different Correlation Models Used Often in the Literature for the Calculation of Effective Thermal Conductivity.

\begin{tabular}{|c|c|c|c|}
\hline Author & Correlations Formulae & $\begin{array}{l}\text { Application } \\
\text { Conditions }\end{array}$ & Ref. \\
\hline Maxwell & $\frac{K_{c}}{K_{p}}=\left[\frac{K_{f}+2 K_{p}+2 V_{f}\left(K_{f}-K_{p}\right)}{K_{f}+2 K_{p}-2 V_{f}\left(K_{f}-K_{p}\right)}\right]$ & $\begin{array}{l}\text { Particle filler low } \\
\text { content }\left(V_{f}<10\right)\end{array}$ & [17] \\
\hline Russell & $\frac{K_{c}}{K_{p}}=\left[\frac{V_{f}^{2 / 3}+\frac{K_{p}}{K_{f}}\left(1-V_{f}^{2 / 3}\right)}{V_{f}^{2 / 3}-V_{f}+\frac{K_{p}}{K_{f}}\left(1-V_{f}^{2 / 3}\right)}\right]$ & $\begin{array}{l}\text { Particle filler low } \\
\text { content }\left(V_{f}<10\right)\end{array}$ & [18] \\
\hline Baschirow-Selenew & $\begin{array}{l}\frac{K_{c}}{K_{p}}=1-\frac{a^{2} \Pi}{4}+\frac{a \Pi p}{2}\left[1-\frac{p}{a} \ln \left(1+\frac{a}{p}\right)\right] \\
\frac{K_{f}}{K_{p}-K_{f}}, a=\left(\frac{6 V_{f}}{\Pi}\right)\end{array}$ & $\begin{array}{l}\text { Particle filler low } \\
\text { content }\left(V_{f}<10\right)\end{array}$ & [19] \\
\hline
\end{tabular}

\section{CONCLUSION}

Finally, we give a brief summary of our results. In this study we have discussed a completely new methodology of enhancing effective thermal conductivity in the composite polymers. We have used polyethylene as the matrix polymer and, aluminium as the metallic filler. We have shown that it is the interface between the "matrix polymer and filler material" which plays a major role in determining the effective thermal conductivity. As the interface, i.e., the contact surface area between the two components of the composite is maximized the thermal conductivity is enhanced. Therefore, without changing the given volume percent of the filler, by maximizing the surface of the filler material (i.e., the interfaces and hence the contact surface area), we can increase the thermal conductivity effectively. This paves a new route of designing composite polymers using the fillers which can maximize the gradient of the heat flux.

There has been a search of best possible fillers in shape and size to increase the effective thermal conductivity in the composite polymers. Our results clearly indicate that those geometrical structures will maximize the conductivity which have a high surface to volume ratio for a given volume percent. To validate this claim we have used different fillers in various shapes and sizes and have shown the respective thermal conductivities. We have used fillers with shapes such as hollow cylinder, solid cylinder, sphere shape, I-shape, $\mathrm{X}$-shape, Y-shape, T-shape, cube, tetrahedron, octahedron etc. to compare the results.
The basic and possible mechanism for the increase in thermal conductivity by surface maximization is due the molecular structure of the polymer itself. Polymers are thermal insulators in general. Unlike in the metals in which the phonons are the carriers of the heat flux, there is no basic unit for carrying the heatflux in the polymers. However, when polymer molecules (which are basically in the form of a chain) come into contact with a metal which has a heat flux, these molecules start local vibrations. When the surface area of the filler material is more, it induces more polymer molecules to vibrate locally and they are coupled locally thereby facilitating more routes for heat flux.

Our results are of the paramount importance in the fabrication of very robust and cost-effective composite polymers which will find high applications in engineering and science. The less use of metal filler in the polymer will make it more light-weight since the density of metallic fillers is very high.

\section{ACKNOWLEDGMENTS}

The simulation work is supported by chemical department of Indian institute of technology, Delhi, India.

\section{REFERENCES}

1. Zhai S, Zhang P, Xian Y, Zeng J, Shi B. Effective Thermal Conductivity of Polymer Composites: Theoretical Models and Simulation Models. Int $J$ Heat Mass Transfer. 2018; 117: 358-374p.

2. Wang X, Liu H, Qiu X, Wang L, Liangbi Wang. Thermal Conductivity of Filled 
Composite Materials Considering Interactions between Fillers. Appl Therm Eng.2018; 141: 835-843p.

3. Wang XJ, Zhang LZ, Pei LX. Thermal Conductivity Augmentation of Composite Polymer Materials with Artificially Controlled Filler Shapes. J Appl Polym Sci. 2013; 131: 39550p.

4. Zhang LZ, Wang XJ, Quan YY, Pei LX. Conjugate Heat Conduction in Filled Composite Materials Considering Interactions between the Filler and Base Materials. Int J Heat Mass Transfer. 2013; 64: 735-742p.

5. Zhang LZ, Wang XJ, Pei LX. Nonlinear Programming Optimization of Filler Shapes for Composite Materials with Inverse Problem Technique to Maximize Heat Conductivity. Int $J$ Heat Mass Transfer.2012; 55(23-24): 7287-7296p.

6. Wang XJ. The Effect of the Prismatic Filler Arrangement and Cross-Sectional Shape on the Thermal Conductivity of Polymer Composites. Express Polym Lett.2014; 8(12): 920-931p.

7. Tong Z, Liu M, Bao H. A Numerical Investigation on the Heat Conduction in High Filler Loading Particulate Composites. Int $J$ Heat Mass Transfer. 2016; 100: 355-361p.

8. Rao Z, Chen B, Zhao J. A Series of Generalized Correlations for Predicting the Thermal Conductivity of Composite Materials Packing with Artificially Designed Filler Shapes. Appl Therm Eng.2017; 120: 444-452p.

9. Hussain ARJ, Alahyari AA, Eastman SA, Erkey CT, Johnston S, Sobkowicz M. Review of Polymers for Heat Exchanger Applications: Factors Concerning Thermal Conductivity. J Appl Therm Eng.2017; 113: $1118-1127 \mathrm{p}$.

10. Oppelt T, Urbaneck T, Böhme H, Platzer B. Numerical Investigation of Effective Thermal Conductivity for Two-Phase Composites Using a Discrete Model. Appl Therm Eng. 2017; 115: 1-8p.

11. Chen X, Su Y, Reay D, Riffat S. Recent Research Developments in Polymer Heat Exchangers: A Review. Renew Sust Energ Rev. 2016; 60: 1367-1386p.

12. Kim HS, Kim JH, Kim WY, Lee HS, Kim SY, Khil MS. Volume Control of
Expanded Graphite Based on Inductively Coupled Plasma and Enhanced Thermal Conductivity of Epoxy Composite by Formation of the Filler Network. Carbon. 2017; 119: 40-46p.

13. GuJ, Yang X, Lv Z, Li N, Liang C, Zhang Q. Functionalized Graphite Nanoplatelets/Epoxy Resin Nanocomposites with High Thermal Conductivity. Int $J$ Heat Mass Transfer.2016; 92: 15-22p.

14. Yang C, Yin XH, Li XP, Zhang Z, Kan JW, Cheng GM. Simulation Study on Flow Dependent Thermal Conductivity of PC/MWCNTs Nanocomposites Considering Interface Topography. Appl Therm Eng.2016; 100(C): 1207-1218p.

15. Agrawal A, Satapathy A. Mathematical Model for Evaluating Effective Thermal Conductivity of Polymer Composites with Hybrid Fillers. Int $J$ Therm Sci.2015; 89(C): 203-209p.

16. Chen H, Ginzburg VV, Yang J, Yang Y, Liu W, Huang Y, Du L, Chen B. Thermal Conductivity of Polymer-Based Composites: Fundamentals and Applications. Prog Polym Sci.2016; 59: 41-85p.

17. Maxwell JC. A Treatise on Electricity and Magnetism: Unabridged. Vol. 2. New York: Dover;1954.

18. Russell HW. Principles of Heat Flow in Porous Insulators. J Am Ceram Soc.1935; 18(1-12): 1-5p

19. Baschirow AB, Manukian AM. Thermal Conductivities of Polymers at Various Temperatures and Pressures. Mech Polim. 1974; 3: 564-566p.

20. Bruggeman DAG. Dielectric Constant and Conductivity of Mixtures of Isotropic Materials. Ann Phys. 1953; 24: 636-669p.

21. Halpin JC. Stiffness and Expansion Estimates for Oriented Short Fiber Composites. J Compos Mater.1969; 3: $732-734 p$.

22. Springer GS, Tsai SW. Thermal Conductivities of Unidirectional Materials. J Compos Mater. 1967; 1(2): 166-173p.

23. Agari Y, Uno T. Estimation on Thermal Conductivities of Filled Polymers. J Appl Polym Sci.1986; 32(7): 5705-5712p.

24. Agari Y, Ueda A, Nagai S. Thermal Conductivity of Apolyethylene Filled with Disoriented Short-Cut Carbon Fibers. $J$ Appl Polym Sci.1991; 43(6): 1117-1124p. 
25. Agari Y, Ueda A, Nagai S. Thermal Conductivities of Composites in Several Types of Dispersion Systems. J Appl Polym Sci.1994; 42: 1665-1669p.

26. Lewis TB, Nielsen LT. Dynamic Mechanical Properties of ParticulateFilled Composites. J Appl Polym Sci. 1970; 14(6): 1449-1471p.

27. NielsenL. The Thermal and Electrical Conductivity of Two-Phase Systems. Ind Eng Chem Fundam.1974; 13(1): 17-20p.

\section{Cite this Article}

Vijendra Kumar, Abhishek Barnwal, R.K. Shukla, Jyoti Shakya. Role of FillerPolymer Interface on the Thermal Conductivity in Polymer Composites. Journal of Polymer \& Composites. 2020; 8(1): 53-70p. 